\title{
Thrombocytopenia in Patients with Chronic Liver Disease: What's in a Name?
}

\author{
Edoardo G. Giannini
}

Published online: 20 October 2012

(c) Springer Science+Business Media New York 2012

Thrombocytopenia is likely the most common haematological alteration that can be observed in patients affected by chronic liver disease [1]. In compensated cirrhosis, it is the most prevalent and incident peripheral blood cytopenia; in chronic hepatitis $\mathrm{C}$ patients, thrombocytopenia represents an obstacle to antiviral therapy in $6.5 \%$ of patients who are otherwise good candidates for interferon treatment $[2,3]$. Furthermore, besides being the hallmark of a possible increased risk of bleeding, thrombocytopenia has several diagnostic and prognostic meanings [4, 5]. This versatile use of platelet count and thrombocytopenia is supported by the multi-faceted etiology of decreased platelet count in chronic liver disease patients [1, 5]. Indeed, the platelet count is incorporated into numerous diagnostic algorithms aimed at non-invasively assessing the severity of chronic liver diseases and features of portal hypertension, can be used to pinpoint patients at higher risk of developing hepatocellular carcinoma in population studies, and is a predictor of death in cirrhotic patients with and without hepatocellular carcinoma [6-12].

The study by Hermos et al. [13] published in this issue of Digestive Diseases and Sciences describes the longitudinal course of platelet count in a large series of patients with non-hepatitis C-related chronic liver disease followed for a median of 3.3 years at the New England Veterans Administration Center, evaluating the occurrence of severe thrombocytopenia-defined as platelet count below $50 \times 10^{9} / \mathrm{L}$ - and significant bleeding, and the association between bleeding episodes and platelet counts. The

E. G. Giannini $(\bowtie)$

Gastroenterology Unit, Department of Internal Medicine, University of Genoa, Viale Benedetto XV, no.6, 16132 Genoa, Italy

e-mail: egiannini@unige.it relevance of this retrospective study lies in its ability to provide reliable data on the time-trend of platelet counts in a large cohort of chronic liver disease patients consistently followed over an adequate period of time, showing that severe thrombocytopenia occurs in a modest proportion of patients $(13.4 \%)$ and is expectedly more incident among patients with a baseline platelet count close to the threshold (i.e., $50 \times 10^{9} / \mathrm{L}$ ). As compared to previous studies that assessed platelet count modifications over time, it has the merit of including patients with all forms of chronic liver disease while excluding patients with chronic hepatitis $\mathrm{C}$ virus infection where multiple causes of thrombocytopenia might have impaired interpretation of the results [14, 15].

The most important information contained in the study, however, is provided by the analyses of the association between platelet counts and bleeding episodes. Indeed, the current vision of the coagulopathy of chronic liver disease patients reflects the presence of a balanced coagulation asset despite altered blood coagulation tests, due to the lack of standardized tests able to adequately evaluate the derangement in both pro- and anti-coagulant factors [16]. Therefore, data regarding a possible association between platelet counts and bleeding might improve our knowledge on this topic. Overall, this study confirms that a decreased platelet count is a good indicator of the severity of chronic liver diseases, as patients with more compromised liver function and higher Model for End-stage Liver Disease (MELD) scores were prevalent in the group of patients with baseline severe thrombocytopenia. Bleeding as a primary cause of hospitalization occurred in $10.8 \%$ of the patients, an incidence five times higher in patients with severe thrombocytopenia compared with patients with a platelet count above the lower limit of normal. Nevertheless, $98.1 \%$ of the bleeding episodes reported in this study were gastrointestinal; unfortunately, no data were available regarding their relation to 
invasive procedures. Interestingly enough, although a strong association between very low platelet counts and bleeding episodes was not identified in the study, the overall mean of minimum platelet count in patients who bled was $76.2 \times 10^{9} / \mathrm{L}$, similar to the platelet count threshold for risk of bleeding following invasive procedures in patients being considered for liver transplantation [4]. As the authors point out, the lack of characterization of the bleeding episodes is a major drawback of the study as it is unlikely that a low platelet count per se might have been a risk factor for bleeding in this patient population. This hypothesis is supported by the low number of central nervous system haemorrhages observed in the study (i.e., 1.5\%) as compared to the increased risk of this type of bleeding in patients with liver disease complicated by immune thrombocytopenia, where very low platelet counts are often observed and thrombocytopenia itself represents a major risk factor for haemorrhage [17]. Taking into consideration the abovementioned limitations of the study, the findings that low platelet counts, anemia, increased INR, and presence of esophageal varices were independently associated with bleeding episodes still leaves us wondering whether thrombocytopenia may just be considered an indirect marker of portal hypertension-related bleeding risk in these patients. This hypothesis is further supported by the important finding that patients attending gastroenterology/hepatology clinics had lower risk for bleeding as compared to patients who did not receive specialized care despite similar platelet counts, thus indirectly suggesting that patients followed at subspecialty centers likely received specific interventions for bleeding prophylaxis.

There are still several open questions that this study was not able to answer. A deeper insight into bleeding episodes might have provided information regarding whether these events had a worse outcome in terms of requirements for transfusions, length of hospital stay, morbidity and death in patients with severe thrombocytopenia as compared with patients with higher platelet counts. As the authors suggest that this population may be the target for thrombopoietic drugs it remains to be established whether simply increasing platelet counts may improve patients outcome, or whether this class of drugs may be helpful for thrombocytopenic patients undergoing invasive procedures alone [18]. Data regarding the severity of features of advanced liver disease (e.g., ascites, hepatic encephalopathy) as well as some relevant laboratory data were missing due to the retrospective study design and therefore important prognostic parameters such as the Child-Pugh score could not be calculated, and its relationship with bleeding episodes not assessed. Lastly, the study results were obtained in a prevalently male population with a high proportion of patients with alcohol dependence or alcohol abuse that was clustered in the group of patients with severe thrombocytopenia, thus preventing generalizability of the study results to populations with different demographic and etiological characteristics. We still need large-scale, prospective studies with adequately collected data to fully evaluate the multiple meanings of thrombocytopenia in patients with chronic liver disease.

Conflict of interest Edoardo G. Giannini was a member of the board and received grant support, consulting fees, lecture fees, and travel reimbursement from GlaxoSmithKline, and participated as an expert witness at a Strategic Advisory Group meeting at the European Medicines Agency concerning eltrombopag for GlaxoSmithKline.

\section{References}

1. Giannini EG. Review article: thrombocytopenia in chronic liver disease and pharmacologic treatment options. Aliment Pharmacol Ther. 2006;23:1055-1065.

2. Qamar AA, Grace ND, Groszmann RJ, et al. Incidence, prevalence, and clinical significance of abnormal hematologic indices in compensated cirrhosis. Clin Gastroenterol Hepatol. 2009;7:689-695.

3. Giannini EG, Marenco S, Fazio V, Pieri G, Savarino V, Picciotto A. Peripheral blood cytopaenia limiting initiation of treatment in chronic hepatitis $\mathrm{C}$ patients otherwise eligible for antiviral therapy. Liver Int. 2012;32:1113-1119.

4. Giannini EG, Greco A, Marenco S, Andorno E, Valente U, Savarino $\mathrm{V}$. Incidence of bleeding following invasive procedures in patients with thrombocytopenia and advanced liver disease. Clin Gastroenterol Hepatol. 2010;8:899-902.

5. Giannini EG, Savarino V. Thrombocytopenia in liver disease. Curr Opin Hematol. 2008;15:473-480.

6. Wai CT, Greenson JK, Fontana RJ, et al. A simple noninvasive index can predict both significant fibrosis and cirrhosis in patients with chronic hepatitis C. Hepatology. 2003;38:518-526.

7. Giannini EG, Zaman A, Ceppa P, Mastracci L, Risso D, Testa R. A simple approach to noninvasively identifying significant fibrosis in chronic hepatitis $\mathrm{C}$ patients in clinical practice. J Clin Gastroenterol. 2006;40:521-527.

8. Giannini EG, Zaman A, Kreil A, et al. Platelet count/spleen diameter ratio for the noninvasive diagnosis of esophageal varices: results of a multicenter, prospective, validation study. Am J Gastroenterol. 2006;101:2511-2519.

9. Burton JR Jr, Liangpunsakul S, Lapidus J, Giannini E, Chalasani N, Zaman A. Validation of a multivariate model predicting presence and size of varices. J Clin Gastroenterol. 2007;41:609-615.

10. Ying L, Lin X, Xie ZL, Hu YP, Shi KQ. Performance of platelet count/spleen diameter ratio for diagnosis of esophageal varices in cirrhosis: a meta-analysis. Dig Dis Sci. 2012;57:1672-1681.

11. D'Amico G, Garcia-Tsao G, Pagliaro L. Natural history and prognostic indicators of survival in cirrhosis: a systematic review of 118 studies. J Hepatol. 2006;44:217-231.

12. Shiina $S$, Tateishi R, Arano T, et al. Radiofrequency ablation for hepatocellular carcinoma: 10-year outcome and prognostic factors. Am J Gastroenterol. 2012;107:569-577.

13. Hermos JA, Altincatal A, Weber HC, et al. Thrombocytopenia and bleeding in Veterans with non-hepatitis C-related chronic liver disease. Dig Dis Sci. (Epub ahead of print). doi: 10.1007/s10620-012-2404-0.

14. Giannini EG, Savarino V. Further insights into the causes of thrombocytopenia in chronic hepatitis C. J Gastrointest Liver Dis. 2010;19:357-358.

15. Giannini EG, Savarino V. Epidemiology of thrombocytopenia in patients with chronic hepatitis C: more than meets the eye. $J$ Viral Hepat. 2011;18:8-10.

16. Tripodi A, Mannucci PM. The coagulopathy of chronic liver disease. N Engl J Med. 2011;365:147-156. 
17. Rajan SK, Espina BM, Liebman HA. Hepatitis C virus-related thrombocytopenia: clinical and laboratory characteristics compared with chronic immune thrombocytopenic purpura. $\mathrm{Br} J$ Haematol. 2005;129:818-824.
18. Afdhal NH, Giannini EG, Tayyab GG, et al. Eltrombopag before procedures in patients with cirrhosis and thrombocytopenia. $N$ Engl J Med. 2012;367:716-724. 